\title{
Avaliação da condição periodontal em pacientes de 10 a 18 anos com diferentes más oclusões
}

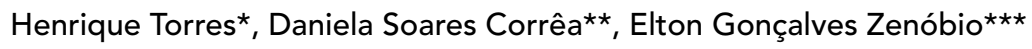

Resumo

Objetivo: o presente trabalho avaliou a condição periodontal de pacientes com idades entre 10 e 18 anos. Metodologia: todos os pacientes eram portadores das seguintes más oclusões: sobressaliência maior que $6 \mathrm{~mm}$, sobremordida maior que $6 \mathrm{~mm}$, sobressaliência e sobremordida associadas maiores que $6 \mathrm{~mm}$ ou mordida cruzada posterior unilateral ou bilateral. Os parâmetros clínicos periodontais (índice de placa, sangramento gengival, mucosa ceratinizada inserida, recessão periodontal, profundidade de sondagem e perda de inserção) foram mensurados em dentes-índices (16, 11, 26, 36, 42 e 46). Os resultados foram analisados pelo teste estatístico de Kruskall Wallis $(\mathrm{p}<0,05)$. Resultados: observou-se que a condição periodontal dos dentes índices, nos quatro grupos de más oclusões avaliadas, apresentou-se nos limites de normalidade. Entretanto, observou-se uma maior tendência ao acúmulo de placa nos pacientes portadores de sobressaliência e sobremordida associadas, bem como nos portadores de mordida cruzada posterior que, também, apresentaram aumento na profundidade de sondagem, no entanto, sem significância estatística. Conclusão: a gravidade da má oclusão não esteve relacionada de maneira significante à presença de doença periodontal.

Palavras-chave: Má oclusão. Doença periodontal.

\section{INTRODUÇÃO}

Tem-se discutido que certos traços morfológicos da má oclusão predispõem ao desenvolvimento da doença periodontal. Estudos ${ }^{3,5,8,11,14}$ buscaram estabelecer a associação entre má oclusão e a doença periodontal, porém resultados conflitantes foram obtidos. Uma associação entre acúmulo de placa, cálculo, gengivite e bolsa periodontal e a má oclusão, segundo a classificação de Angle, foi sugerida; entretanto, essa associação não tem se confirmado. Poulton e Aaronson ${ }^{15}$ afirmaram existir uma relação estatisticamente signi- ficante entre oclusão e saúde periodontal; porém a demonstração desta correlação não comprova a existência de uma relação de causa e efeito entre os fatores envolvidos.

A presença do apinhamento dentário tem atraído atenção especial. Poulton, Aaronson ${ }^{15}$ e Addy et al. ${ }^{1}$ encontraram um risco aumentado para o desenvolvimento de gengivite e bolsa periodontal em pacientes com mau posicionamento dentário e falta de espaço no arco. No entanto, nos casos com bom nível de higiene bucal e também nos casos com controle de placa deficiente, a associação entre

* Especialista e Mestre em Ortodontia pela PUC-MG. Professor Assistente do Curso de Mestrado em Ortodontia PUC-MG.

** Especialista em Periodontia pela PUC-MG. Mestre em Clínicas Odontológicas - Área de concentração em Periodontia pela PUC-MG.

*** Doutor e Mestre em Periodontia pela UNESP/Araraquara. Coordenador e Professor da Área de Concentração em Periodontia do Mestrado em Clínicas Odontológicas e Ortodontia da PUC/MG. Professor Responsável da Disciplina de Periodontia da FOUI/MG. 
apinhamento e doença periodontal é duvidosa ${ }^{2}$.

A relação entre doença periodontal e a sobressaliência e sobremordida exageradas também tem sido proposta. Alexander e Tipnis ${ }^{3}$ não encontraram associação entre sobressaliência, sobremordida e doença periodontal. Geiger, Wasserman e Turgeon ${ }^{8}$ salientaram que a sobressaliência, na maioria das vezes, não está relacionada à destruição periodontal, mas acreditam que possivelmente a sua manifestação, na forma mais grave, pode contribuir para o progresso da doença periodontal. Helm e Petersen ${ }^{11}$ relataram que a sobremordida de maior magnitude $(6 \mathrm{~mm})$ e a mordida cruzada podem aumentar o risco de doença periodontal. Bjornas, Rygh e $\mathrm{Boe}^{5}$ consideraram que as investigações prévias que buscaram relacionar sobressaliência e doença periodontal não encontraram uma correlação positiva porque observaram poucas más oclusões de grande severidade.

Os achados contraditórios na literatura podem, em parte, ser explicados pela utilização de métodos diferentes de avaliação da má oclusão e da doença periodontal. Portanto, estudos padronizados devem ser realizados com o objetivo de relacionar a associação da má oclusão à doença periodontal.

O presente estudo se propôs a:

1) Avaliar clinicamente a presença de uma associação entre traços de má oclusão definidos (sobressaliência e/ou sobremordida maior que $6 \mathrm{~mm}$ e mordida cruzada posterior) e condição periodontal, em pacientes de 10 a 18 anos.

2) Avaliar as diferenças, nas condições periodontais, entre os grupos de más oclusões (sobressaliência e/ou sobremordida maior que $6 \mathrm{~mm}$ e mordida cruzada posterior).

\section{METODOLOGIA}

Foram examinados clinicamente 245 indivíduos, com idades de 10 a 18 anos, inscritos para tratamento ortodôntico na clínica de Ortodontia do curso de Mestrado em Odontologia da Pontifícia Universidade Católica de Minas Gerais. Dessa população, foram selecionados 49 indivíduos que apresentavam um dos seguintes traços morfológicos de má oclusão: sobressaliência maior que $6 \mathrm{~mm}$ e/ou sobremordida maior que $6 \mathrm{~mm}$, ou mordida cruzada posterior.

Os critérios de exclusão utilizados na seleção dos pacientes foram: presença de doenças sistêmicas, pacientes em uso de certos medicamentos (ex. Dilantin), presença de aparelhos fixos ou removíveis, presença de próteses, ausência de um dos dentes-índices $(16,26,36,46,11$ e 42) ou pacientes fumantes.

Dos 49 indivíduos examinados, 8 foram excluídos do estudo devido à presença de aparelho ortodôntico fixo parcial ( $\mathrm{n}=2)$; ausência do elemento 16 $(n=1)$; ausência do elemento $46(n=1)$; não comparecimento à consulta para exame periodontal $(\mathrm{n}=2)$; presença das três más oclusões simultaneamente $(n=2)$. Da população final de 41 pacientes selecionados para a pesquisa, 12 indivíduos possuíam sobressaliência maior que $6 \mathrm{~mm}(29,3 \%), 5$ indivíduos possuíam sobremordida maior que $6 \mathrm{~mm}$ $(12,2 \%), 11$ indivíduos possuíam as duas características anteriores associadas $(26,8 \%)$ e 13 indivíduos possuíam mordida cruzada posterior $(31,7 \%)$, sendo $30,8 \%$ bilaterais e $69,2 \%$ unilaterais.

Esta amostra foi submetida à avaliação da condição periodontal, a partir dos dentes-índices 16, 26, 36, 46, 11 e 42, utilizando os seguintes

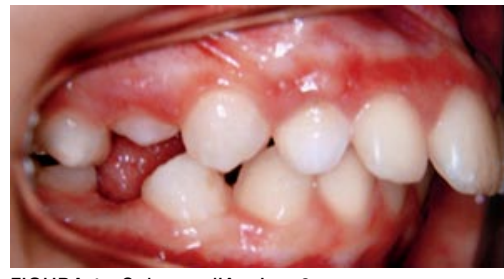

FIGURA 1 - Sobressaliência > 6mm.

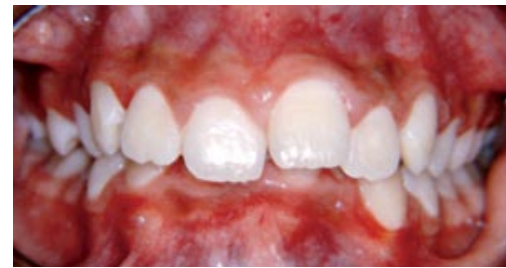

FIGURA 2 - Sobremordida $>6 \mathrm{~mm}$.

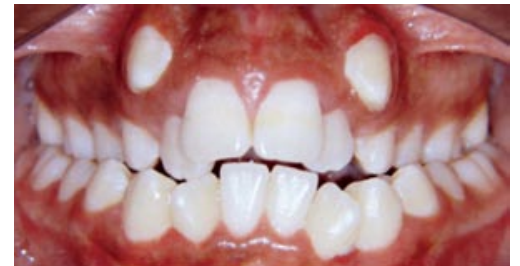

FIGURA 3 - Mordida cruzada posterior. 
parâmetros: índice de placa (Quigley, Hein ${ }^{16}, 1962$, modificado); índice de sangramento gengival ${ }^{13}$; profundidade de sondagem, quantidade de mucosa ceratinizada inserida, recessão gengival e perda de inserção.

\section{RESULTADOS}

Com relação ao índice de placa, observou-se que no grupo com mordida cruzada posterior houve $46,2 \%$ dos casos com $1 / 3$ das superfícies dentárias recobertas por placa e $53,8 \%$ com $2 / 3$ dos dentes afetados. No grupo com sobressaliência e sobremordida, observou-se $63,6 \%$ dos pacientes com $1 / 3$ dos dentes afetados e $36,4 \%$ com $2 / 3$ dos dentes afetados. Portanto, constatou-se uma tendência a um maior acúmulo de placa associado à

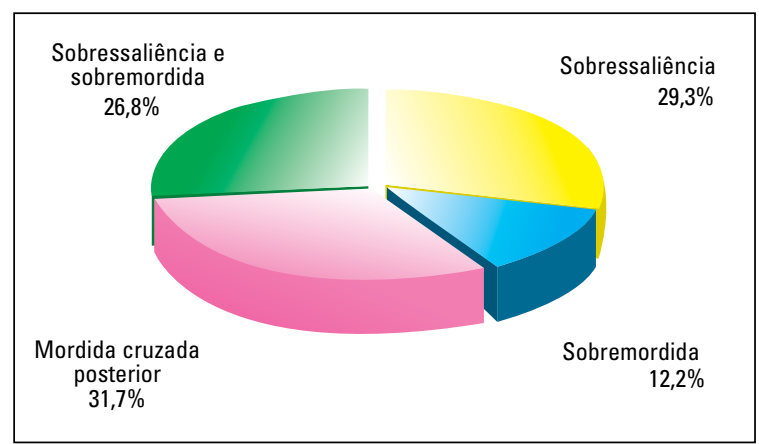

GRÁFICO 1 - Distribuição dos pacientes da amostra, de acordo com o tipo de má oclusão.

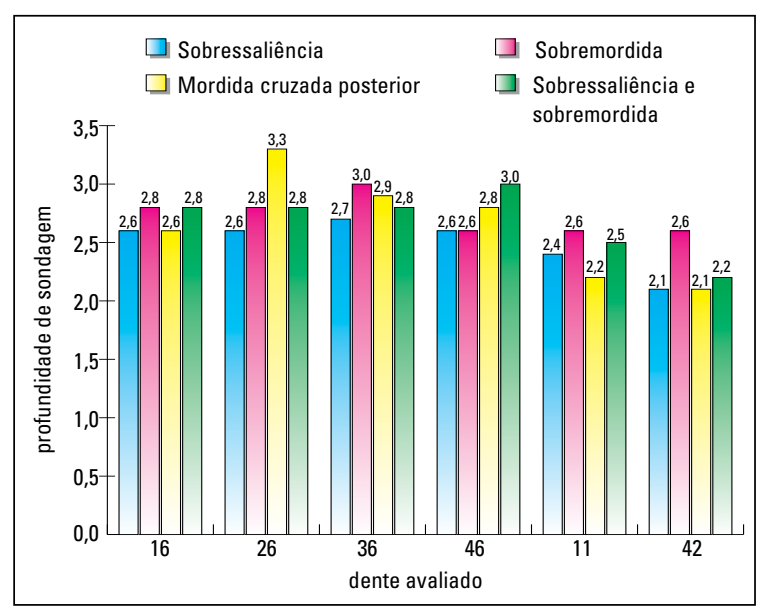

GRÁFICO 2 - Caracterização dos pacientes, segundo a profundidade de sondagem. má oclusão nos pacientes portadores de mordida cruzada posterior e nos portadores de sobressaliência e sobremordida associadas.

Quanto ao índice de sangramento gengival, não foram constatadas diferenças significativas entre os quatro grupos estudados (Tab. 1). Os valores médios encontrados em todos os grupos, entre $0 \mathrm{e}$ 1, estão dentro dos limites aceitos como normalidade do periodonto.

Em relação à profundidade de sondagem, também não foram verificadas diferenças significativas entre os grupos pesquisados, independentemente do dente avaliado. Os valores médios encontrados (menores que $3 \mathrm{~mm}$ ) para cada dente, nos quatro tipos de má oclusão, são compatíveis com um periodonto saudável. O dente-índice 26 , nos pacientes com mordida cruzada posterior, apresentou um valor médio $(3,3 \mathrm{~mm})$ e um desvio padrão $(1,5 \mathrm{~mm})$ um pouco acima da média (Tab. 2, Gráf. 2).

Os valores médios de mucosa ceratinizada inserida de todos os dentes-índices nos quatro grupos de má oclusão foram sempre compatíveis com um periodonto saudável, com medida superior a $1 \mathrm{~mm}$, em todos os dentes avaliados e em todos os grupos de más oclusões (Tab. 3). Comparando-se os grupos, foram observadas diferenças significativas apenas no dente 11 . Neste caso, constataramse valores menores no grupo com mordida cruzada posterior, quando comparado com os demais, que não diferiram entre si.

Em relação à recessão periodontal, os valores

Tabela 1 - Comparação entre os grupos quanto ao índice de sangramento gengival.

\begin{tabular}{ccccccc}
\hline \multirow{2}{*}{ grupo } & \multicolumn{7}{c}{ medidas descritivas } \\
\cline { 2 - 7 } & mínimo & máximo & mediana & média & desvio & $\mathbf{p}$ \\
\hline 1 & 0 & 1 & 1 & 0,8 & 0,4 & \\
2 & 0 & 1 & 0 & 0,2 & 0,4 & 0,141 \\
3 & 0 & 1 & 0 & 0,6 & 0,5 & $1=2=4$ \\
4 & 0 & 2 & 1 & 0,8 & 0,7 & \\
\hline
\end{tabular}

Nota: A probabilidade de significância refere-se ao teste Kruskal-Wallis. Grupo 1 = Sobressaliência; Grupo 2 = Sobremordida; Grupo 3 = Mordida Cruzada Posterior; Grupo 4 = Sobressaliência + Sobremordida. 
Tabela 2 - Caracterização dos pacientes, segundo a profundidade de sondagem.

\begin{tabular}{|c|c|c|c|c|c|c|c|}
\hline \multirow{2}{*}{$\begin{array}{c}\text { den- } \\
\text { te }\end{array}$} & \multirow{2}{*}{$\begin{array}{c}\text { gru- } \\
\text { po }\end{array}$} & \multicolumn{5}{|c|}{ medidas descritivas } & \multirow[b]{2}{*}{$\mathbf{p}$} \\
\hline & & $\begin{array}{c}\text { míni- } \\
\text { mo }\end{array}$ & $\begin{array}{c}\text { máxi- } \\
\text { mo }\end{array}$ & mediana & média & desvio & \\
\hline \multirow{4}{*}{16} & 1 & 2 & 3 & 3 & 2,6 & 0,5 & \multirow{4}{*}{$\begin{array}{c}0,736 \\
1=2=3=4\end{array}$} \\
\hline & 2 & 2 & 3 & 3 & 2,8 & 0,4 & \\
\hline & 3 & 1 & 5 & 2 & 2,6 & 1,1 & \\
\hline & 4 & 2 & 5 & 3 & 2,8 & 0,9 & \\
\hline \multirow{4}{*}{26} & 1 & 2 & 4 & 2,5 & 2,6 & 0,7 & \multirow{4}{*}{$\begin{array}{c}0,337 \\
1=2=3=4\end{array}$} \\
\hline & 2 & 2 & 3 & 3 & 2,8 & 0,4 & \\
\hline & 3 & 1 & 5 & 3 & 3,3 & 1,3 & \\
\hline & 4 & 2 & 7 & 2 & 2,8 & 1,5 & \\
\hline \multirow{4}{*}{36} & 1 & 2 & 4 & 2,5 & 2,7 & 0,8 & \multirow{4}{*}{$\begin{array}{c}0,685 \\
1=2=3=4\end{array}$} \\
\hline & 2 & 3 & 3 & 3 & 3 & 0 & \\
\hline & 3 & 1 & 5 & 3 & 2,9 & 1,1 & \\
\hline & 4 & 2 & 5 & 3 & 2,8 & 0,9 & \\
\hline \multirow{4}{*}{46} & 1 & 2 & 4 & 2 & 2,6 & 0,8 & \multirow{4}{*}{$\begin{array}{c}0,520 \\
1=2=3=4\end{array}$} \\
\hline & 2 & 1 & 3 & 3 & 2,6 & 0,9 & \\
\hline & 3 & 1 & 4 & 3 & 2,8 & 0,8 & \\
\hline & 4 & 2 & 5 & 3 & 3 & 0,8 & \\
\hline \multirow{4}{*}{11} & 1 & 2 & 4 & 2 & 2,4 & 0,7 & \multirow{4}{*}{$\begin{array}{c}0,729 \\
1=2=3=4\end{array}$} \\
\hline & 2 & 2 & 3 & 3 & 2,6 & 0,5 & \\
\hline & 3 & 1 & 3 & 2 & 2,2 & 0,7 & \\
\hline & 4 & 2 & 3 & 2 & 2,5 & 0,5 & \\
\hline \multirow{4}{*}{42} & 1 & 1 & 4 & 2 & 2,1 & 0,9 & \multirow{4}{*}{$\begin{array}{c}0,525 \\
1=2=3=4\end{array}$} \\
\hline & 2 & 2 & 3 & 3 & 2,6 & 0,5 & \\
\hline & 3 & 1 & 3 & 2 & 2,1 & 0,8 & \\
\hline & 4 & 1 & 3 & 2 & 2,2 & 0,8 & \\
\hline
\end{tabular}

Nota: A probabilidade de significância refere-se ao teste Kruskal-Wallis. Grupo 1 = Sobressaliência; Grupo 2 = Sobremordida; Grupo 3 = Mordida Cruzada Posterior; Grupo 4 = Sobressaliência + Sobremordida.

médios para todos os dentes-índices nos quatro grupos de más oclusões foram, em mais de $97 \%$ dos casos, iguais a 0 , caracterizando ausência dessa condição periodontal (Tab. 4).

Os valores médios de perda de inserção nos quatro grupos pesquisados foram sempre inferiores a $3 \mathrm{~mm}$, estando dentro dos limites aceitos como normalidade do periodonto. Nenhuma diferença significativa entre os grupos foi constatada.
Tabela 3 - Caracterização dos pacientes, segundo a mucosa ceratinizada inserida.

\begin{tabular}{|c|c|c|c|c|c|c|c|}
\hline \multirow{2}{*}{$\begin{array}{l}\text { den- } \\
\text { te }\end{array}$} & \multirow{2}{*}{$\begin{array}{c}\text { gru- } \\
\text { po }\end{array}$} & \multicolumn{5}{|c|}{ medidas descritivas } & \multirow[b]{2}{*}{$\mathbf{p}$} \\
\hline & & $\begin{array}{c}\text { míni- } \\
\text { mo }\end{array}$ & $\begin{array}{c}\text { máxi- } \\
\text { mo }\end{array}$ & $\begin{array}{l}\text { me- } \\
\text { diana }\end{array}$ & $\begin{array}{l}\text { mé- } \\
\text { dia }\end{array}$ & $\begin{array}{c}\text { des- } \\
\text { vio }\end{array}$ & \\
\hline \multirow{4}{*}{16} & 1 & 3 & 5 & 4 & 3,8 & 0,8 & \multirow{4}{*}{$\begin{array}{c}0,490 \\
1=2=3=4\end{array}$} \\
\hline & 2 & 4 & 6 & 4 & 4,4 & 0,9 & \\
\hline & 3 & 2 & 5 & 4 & 3,7 & 1,0 & \\
\hline & 4 & 3 & 6 & 4 & 4,1 & 0,8 & \\
\hline \multirow{4}{*}{26} & 1 & 2 & 5 & 3,5 & 3,7 & 1,2 & \multirow{4}{*}{$\begin{array}{c}0,484 \\
1=2=3=4\end{array}$} \\
\hline & 2 & 4 & 4 & 4 & 4 & 0 & \\
\hline & 3 & 2 & 5 & 4 & 3,8 & 0,9 & \\
\hline & 4 & 3 & 6 & 4 & 4,4 & 1 & \\
\hline \multirow{4}{*}{36} & 1 & 1 & 4 & 3 & 2,7 & 1,1 & \multirow{4}{*}{$\begin{array}{c}0,216 \\
1=2=3=4\end{array}$} \\
\hline & 2 & 2 & 4 & 3 & 3 & 0,7 & \\
\hline & 3 & 2 & 4 & 3 & 3,1 & 0,5 & \\
\hline & 4 & 2 & 3 & 3 & 2,5 & 0,5 & \\
\hline \multirow{4}{*}{46} & 1 & 2 & 3 & 3 & 2,8 & 0,5 & \multirow{4}{*}{$\begin{array}{c}0,628 \\
1=2=3=4\end{array}$} \\
\hline & 2 & 2 & 4 & 3 & 3 & 0,7 & \\
\hline & 3 & 2 & 3 & 3 & 3,2 & 0,5 & \\
\hline & 4 & 2 & 4 & 3 & 4,1 & 0,6 & \\
\hline \multirow{4}{*}{11} & 1 & 3 & 5 & 4 & 4,2 & 0,8 & \multirow{4}{*}{$\begin{array}{c}0,021 \\
1=2=3=4\end{array}$} \\
\hline & 2 & 3 & 5 & 4 & 3,8 & 0,8 & \\
\hline & 3 & 2 & 4 & 3 & 3,2 & 0,7 & \\
\hline & 4 & 3 & 5 & 4 & 4,1 & 0,8 & \\
\hline \multirow{4}{*}{42} & 1 & 2 & 5 & 4 & 3,6 & 1,1 & \multirow{4}{*}{$\begin{array}{c}0,136 \\
1=2=3=4\end{array}$} \\
\hline & 2 & 2 & 6 & 5 & 4,4 & 1,5 & \\
\hline & 3 & 2 & 5 & 3 & 2,9 & 1 & \\
\hline & 4 & 2 & 5 & 4 & 3,5 & 1,2 & \\
\hline
\end{tabular}

Nota: A probabilidade de significância refere-se ao teste Kruskal-Wallis. Grupo 1 = Sobressaliência; Grupo 2 = Sobremordida; Grupo 3 = Mordida Cruzada Posterior; Grupo 4 = Sobressaliência + Sobremordida.

No entanto, no grupo de sobremordida (grupo 2), observou-se valores médios maiores para todos os dentes-índices (Tab. 5).

Comparando-se elementos dentários cruzados e elementos em oclusão normal, utilizando-se os dentes 16/46 em oclusão normal e os dentes 26/36 do grupo de pacientes com mordida cruzada posterior unilateral (9 pacientes), observaram-se diferenças significativas com relação à profundidade de 
Tabela 4 - Caracterização dos pacientes, segundo a recessão periodontal.

\begin{tabular}{|c|c|c|c|c|c|c|c|c|}
\hline \multirow{3}{*}{$\begin{array}{c}\text { den- } \\
\text { te }\end{array}$} & \multirow{3}{*}{$\begin{array}{c}\text { gru- } \\
\text { po }\end{array}$} & \multicolumn{6}{|c|}{ recessão periodontal } & \multirow{3}{*}{ total } \\
\hline & & \multicolumn{2}{|c|}{$0 \mathrm{~mm}$} & \multicolumn{2}{|c|}{$1 \mathrm{~mm}$} & \multicolumn{2}{|c|}{$2 \mathrm{~mm}$} & \\
\hline & & $\mathbf{n}$ & $\%$ & $\mathbf{n}$ & $\%$ & $\mathbf{n}$ & $\%$ & \\
\hline \multirow{4}{*}{16} & 1 & 12 & 100 & 0 & 0 & 0 & 0 & 12 \\
\hline & 2 & 5 & 100 & 0 & 0 & 0 & 0 & 5 \\
\hline & 3 & 13 & 100 & 0 & 0 & 0 & 0 & 13 \\
\hline & 4 & 11 & 100 & 0 & 0 & 0 & 0 & 11 \\
\hline \multirow{4}{*}{26} & 1 & 11 & 91,7 & 1 & 8,3 & 0 & 0 & 12 \\
\hline & 2 & 5 & 100 & 0 & 0 & 0 & 0 & 5 \\
\hline & 3 & 12 & 92,3 & 0 & 0 & 1 & 7,7 & 13 \\
\hline & 4 & 11 & 100 & 0 & 0 & 0 & 0 & 11 \\
\hline \multirow{4}{*}{36} & 1 & 12 & 100 & 0 & 0 & 0 & 0 & 12 \\
\hline & 2 & 5 & 100 & 0 & 0 & 0 & 0 & 5 \\
\hline & 3 & 13 & 100 & 0 & 0 & 0 & 0 & 13 \\
\hline & 4 & 11 & 100 & 0 & 0 & 0 & 0 & 11 \\
\hline \multirow{4}{*}{46} & 1 & 11 & 91,7 & 1 & 8,3 & 0 & 0 & 12 \\
\hline & 2 & 5 & 100 & 0 & 0 & 0 & 0 & 5 \\
\hline & 3 & 12 & 92,3 & 1 & 7,7 & 0 & 0 & 13 \\
\hline & 4 & 11 & 100 & 0 & 0 & 0 & 0 & 11 \\
\hline \multirow{4}{*}{11} & 1 & 12 & 100 & 0 & 0 & 0 & 0 & 12 \\
\hline & 2 & 5 & 100 & 0 & 0 & 0 & 0 & 5 \\
\hline & 3 & 12 & 92,3 & 1 & 7,7 & 0 & 0 & 13 \\
\hline & 4 & 11 & 100 & 0 & 0 & 0 & 0 & 11 \\
\hline \multirow{4}{*}{42} & 1 & 12 & 100 & 0 & 0 & 0 & 0 & 12 \\
\hline & 2 & 5 & 100 & 0 & 0 & 0 & 0 & 5 \\
\hline & 3 & 13 & 100 & 0 & 0 & 0 & 0 & 13 \\
\hline & 4 & 11 & 100 & 0 & 0 & 0 & 0 & 11 \\
\hline
\end{tabular}

Grupo 1 = Sobressaliência; Grupo 2 = Sobremordida; Grupo 3 = Mordida Cruzada Posterior; Grupo 4 = Sobressaliência + Sobremordida.

sondagem; nos quais os dentes 16/46 apresentaram valores inferiores. Quanto à mucosa ceratinizada inserida e à perda de inserção, não se constataram diferenças significativas. Já no grupo de pacientes com mordida cruzada posterior bilateral, não se observaram diferenças significativas entre os lados direito e esquerdo. Estes resultados podem ser observados nas tabelas 6 e 7 .
Tabela 5 - Caracterização dos pacientes, segundo a perda de inserção.

\begin{tabular}{|c|c|c|c|c|c|c|c|}
\hline \multirow[b]{2}{*}{ dente } & \multirow[b]{2}{*}{$\begin{array}{c}\text { gru- } \\
\text { po }\end{array}$} & \multicolumn{5}{|c|}{ medidas descritivas } & \multirow[b]{2}{*}{$\mathbf{p}$} \\
\hline & & $\begin{array}{c}\text { míni- } \\
\text { mo }\end{array}$ & $\begin{array}{c}\text { máxi- } \\
\text { mo }\end{array}$ & $\begin{array}{c}\text { me- } \\
\text { dia- } \\
\text { na }\end{array}$ & $\begin{array}{l}\text { mé- } \\
\text { dia }\end{array}$ & desvio & \\
\hline \multirow{4}{*}{16} & 1 & 0 & 3 & 0 & 1,1 & 1,4 & \multirow{4}{*}{$\begin{array}{c}0,074 \\
1=2=3=4\end{array}$} \\
\hline & 2 & 0 & 3 & 3 & 2,2 & 1,3 & \\
\hline & 3 & 0 & 2 & 0 & 0,4 & 0,8 & \\
\hline & 4 & 0 & 3 & 0 & 1,1 & 1,4 & \\
\hline \multirow{4}{*}{26} & 1 & 0 & 3 & 0 & 1,2 & 1,5 & \multirow{4}{*}{$\begin{array}{c}0,168 \\
1=2=3=4\end{array}$} \\
\hline & 2 & 0 & 3 & 3 & 2,2 & 1,3 & \\
\hline & 3 & 0 & 3 & 0 & 0,5 & 1,1 & \\
\hline & 4 & 0 & 3 & 0 & 1,1 & 1,4 & \\
\hline \multirow{4}{*}{36} & 1 & 0 & 3 & 0 & 0,9 & 1,2 & \multirow{4}{*}{$\begin{array}{c}0,104 \\
1=2=3=4\end{array}$} \\
\hline & 2 & 0 & 3 & 3 & 2,4 & 1,3 & \\
\hline & 3 & 0 & 3 & 0 & 0,7 & 1 & \\
\hline & 4 & 0 & 3 & 0 & 1,1 & 1,4 & \\
\hline \multirow{4}{*}{46} & 1 & 0 & 3 & 0 & 1 & 1,3 & \multirow{4}{*}{$\begin{array}{c}0,316 \\
1=2=3=4\end{array}$} \\
\hline & 2 & 0 & 3 & 3 & 2,0 & 1,4 & \\
\hline & 3 & 0 & 3 & 0 & 0,8 & 1,3 & \\
\hline & 4 & 0 & 3 & 0 & 1,3 & 1,5 & \\
\hline \multirow{4}{*}{11} & 1 & 0 & 4 & 1 & 1,3 & 1,4 & \multirow{4}{*}{$\begin{array}{c}0,289 \\
1=2=3=4\end{array}$} \\
\hline & 2 & 0 & 3 & 2 & 2 & 1,2 & \\
\hline & 3 & 0 & 3 & 0 & 0,8 & 1,1 & \\
\hline & 4 & 0 & 3 & 0 & 1 & 1,2 & \\
\hline \multirow{4}{*}{42} & 1 & 0 & 2 & 0,5 & 0,8 & 0,9 & \multirow{4}{*}{$\begin{array}{c}0,240 \\
1=2=3=4\end{array}$} \\
\hline & 2 & 0 & 3 & 2 & 1,8 & 1,1 & \\
\hline & 3 & 0 & 2 & 0 & 0,6 & 0,8 & \\
\hline & 4 & 0 & 3 & 0 & 0,9 & 1,2 & \\
\hline
\end{tabular}

Nota: A probabilidade de significância refere-se ao teste Kruskal-Wallis. Grupo 1 = Sobressaliência; Grupo 2 = Sobremordida; Grupo 3 = Mordida Cruzada Posterior; Grupo 4 = Sobressaliência + Sobremordida.

\section{DISCUSSÃO}

$\mathrm{Na}$ literatura observam-se relatos de prevalência dos diversos traços de má oclusão das populações estudadas. Geiger ${ }^{7}$ registrou $6 \%$ de pacientes portadores de sobremordida $>6 \mathrm{~mm}$ e $11 \%$ de portadores de sobressaliência $>6 \mathrm{~mm}$. De uma amostra de 516 pacientes, Geiger, Wasserman e Turgeon $^{8}$ registraram $8,7 \%$ de pacientes portadores de 
Tabela 6 - Caracterização dos pacientes com mordida cruzada posterior unilateral, segundo as medidas de interesse, considerando-se os dentes 16/46 e $26 / 36$.

\begin{tabular}{|c|c|c|c|c|c|c|c|}
\hline \multirow{2}{*}{ medida } & \multirow{2}{*}{ dente } & \multicolumn{5}{|c|}{ medidas descritivas } & \multirow{2}{*}{$\mathbf{p}$} \\
\hline & & mínimo & máximo & mediana & média & desvio & \\
\hline \multirow{2}{*}{ PS } & $16 / 46$ & 5 & 5 & 3 & 3,1 & 0,9 & \multirow{2}{*}{$\begin{array}{l}0,046 \\
D_{1}<D_{2}\end{array}$} \\
\hline & $26 / 36$ & 2 & 5 & 3 & 3,6 & 1 & \\
\hline \multirow{2}{*}{$\mathrm{MCl}$} & $16 / 46$ & 2 & 5 & 4 & 3,4 & 1 & \multirow{2}{*}{$\begin{array}{l}0,180 \\
D_{1}=D_{2}\end{array}$} \\
\hline & $26 / 36$ & 3 & 5 & 4 & 3,9 & 0,8 & \\
\hline \multirow{2}{*}{ PI } & $16 / 46$ & 0 & 3 & 0 & 0,3 & 1 & \multirow{2}{*}{$\begin{array}{l}0,307 \\
D_{1}=D_{2}\end{array}$} \\
\hline & $26 / 36$ & 0 & 3 & 0 & 0,4 & 1 & \\
\hline
\end{tabular}

Nota: A probabilidade de significância refere-se ao teste Friedman.

$D_{1} \rightarrow$ dentes $16 / 46 ; D_{2}->$ dentes $26 / 36$.

Tabela 7 - Caracterização dos pacientes com mordida cruzada posterior bilateral, segundo as medidas de interesse, considerando-se os dentes 16/46 e 26/36.

\begin{tabular}{|c|c|c|c|c|c|c|c|}
\hline \multirow{2}{*}{ medida } & \multirow{2}{*}{ dente } & \multicolumn{5}{|c|}{ medidas descritivas } & \multirow{2}{*}{$\mathbf{p}$} \\
\hline & & mínimo & máximo & mediana & média & desvio & \\
\hline \multirow{2}{*}{ PS } & $16 / 46$ & 1 & 4 & 3 & 2,8 & 1,3 & \multirow{2}{*}{$\begin{array}{l}0,157 \\
D_{1}=D_{2}\end{array}$} \\
\hline & $26 / 36$ & 1 & 5 & 3,5 & 3,3 & 1,7 & \\
\hline \multirow{2}{*}{$\mathrm{MCl}$} & $16 / 46$ & 3 & 5 & 4,5 & 4,3 & 1,0 & \multirow{2}{*}{$\begin{array}{l}0,157 \\
D_{1}=D_{2}\end{array}$} \\
\hline & $26 / 36$ & 2 & 5 & 4 & 3,8 & 1,3 & \\
\hline \multirow{2}{*}{ PI } & $16 / 46$ & 0 & 3 & 2 & 1,8 & 1,5 & \multirow{2}{*}{$\begin{array}{c}1 \\
D_{1}=D_{2}\end{array}$} \\
\hline & $26 / 36$ & 0 & 3 & 2 & 1,8 & 1,5 & \\
\hline
\end{tabular}

Nota: A probabilidade de significância refere-se ao teste Friedman.

$D_{1} \rightarrow$ dentes $16 / 46 ; D_{2} \rightarrow$ dentes $26 / 36$.

sobressaliência $>6 \mathrm{~mm}$ e $7 \%$ de pacientes portadores de sobremordida $>6 \mathrm{~mm}$. Alexander e Tipnis ${ }^{3}$ registraram $2 \%$ de sobressaliência $>6 \mathrm{~mm}$ e $16 \%$ de portadores de sobremordida entre 5 e $6 \mathrm{~mm}$, em uma amostra de 50 pacientes, e Helm e Petersen $^{11}$ encontraram $17 \%$ de portadores de sobressaliência $>6 \mathrm{~mm}$ e $9 \%$ de sobremordida $>6 \mathrm{~mm}$. Essa pesquisa constatou que, de uma população de 245 indivíduos, 3\% apresentaram sobremordida $>6 \mathrm{~mm}, 7 \%$ apresentaram sobressaliência $>6 \mathrm{~mm}$ e $6 \%$ apresentaram as duas condições associadas, semelhante aos estudos prévios.

Gould e Picton ${ }^{10}$ relataram uma maior tendência à destruição periodontal em pacientes do gênero masculino, em comparação ao gênero feminino, relacionada à má oclusão. Geiger, Wasserman e Turgeon ${ }^{8}$ observaram que não existe diferença apreciável na distribuição de inflamação gengival em relação ao gênero. Entretanto relataram diferença entre os gêneros na distribuição de doença periodontal severa, que foi predominante no gênero masculino. No presente trabalho, a sobressaliência exagerada esteve presente em $8 \%$ dos homens e em $6 \%$ das mulheres, a sobremordida exagerada em $6 \%$ dos homens e $7 \%$ das mulheres e a mordida cruzada posterior em $4 \%$ dos homens e $6 \%$ das mulheres, com distribuição semelhante.

Dois trabalhos ${ }^{3,12}$ buscaram relacionar o índice de placa à presença de sobressaliência, sobremordida e mordida cruzada. Alexander e Tipnis ${ }^{3}$ encontraram um maior índice de placa nos dentes anteriores inferiores de pacientes portadores de so- 
bressaliência e/ou sobremordida exagerada, quando comparados a pacientes normais. Esta mesma correlação não foi observada por Hörup, Melsen e Terp ${ }^{12}$. A análise do índice de placa no presente trabalho revelou que os pacientes portadores de sobremordida exagerada apresentaram um padrão de normalidade, ao passo que $33,3 \%$ dos portadores de sobressaliência exagerada apresentaram mais de $2 / 3$ do dentes índices recobertos por placa. Nos pacientes com mordida cruzada posterior, $53,8 \%$ apresentaram índice de placa acima do desejável. Já no grupo de pacientes, portadores de sobressaliência e sobremordida associadas, $36,4 \%$ apresentaram essa condição. Acredita-se que este fato se deva à maior dificuldade de higienização dos dentes anteriores superiores, que apresentam excessiva inclinação vestibular nos pacientes portadores de sobressaliência exagerada, principalmente em se tratando de pacientes jovens, menos conscientes quanto à necessidade de uma boa higiene bucal. $\mathrm{O}$ mesmo pode ter ocorrido com os pacientes portadores de mordida cruzada posterior, nos quais os elementos cruzados também exigem maiores cuidados para um bom controle de placa, principalmente quando somente um elemento se encontra nesta condição.

Buckley $^{6}$ avaliou a condição periodontal de pacientes portadores de apinhamento, sobremordida e sobressaliência exagerada, utilizando o índice de Russel, que verifica a presença de inflamação gengival à profundidade de sondagem. Segundo o autor, os pacientes com apinhamento apresentaram inflamação gengival e sinais de destruição periodontal, o que não foi observado nos portadores de sobremordida e sobressaliência. Gould e Picton ${ }^{10}$, entretanto, observaram, em alguns pacientes com sobremordida e sobressaliência, profundidades de sondagem indicativas de destruição periodontal (acima de $3 \mathrm{~mm}$ ). Além disto, a média de profundidade de sondagem dos portadores destas más oclusões foi superior à de pacientes sem má oclusão. Helm e Petersen ${ }^{11}$ observaram maiores índices de gengivite e maior profundidade de sondagem em pacientes com sobressaliência $>6 \mathrm{~mm}$. Não pôde ser detectado um aumento destes índices proporcional ao aumento da gravidade desta má oclusão. Em pacientes com sobremordida $>6 \mathrm{~mm}$ ou com mordida cruzada, não se observaram diferenças significativas em relação aos pacientes sem má oclusão. $\mathrm{O}$ atual estudo apresentou uma média de profundidade de sondagem para todos os dentes examinados, nos quatro grupos de má oclusão, inferior a $3 \mathrm{~mm}$, valor considerado aceitável em pacientes sem doença periodontal. Entretanto, ainda que dentro dos parâmetros normais, as maiores médias $(3,3 \mathrm{~mm})$ foram encontradas no elemento 26 , quando estava presente a mordida cruzada posterior. Acreditamos que a incorreta relação buco-lingual dos molares, que dificulta a auto-limpeza realizada pela mucosa e o adequado controle de placa feito pelo paciente, possam responder por esta tendência. A profundidade de sondagem, dos elementos $11 \mathrm{e}$ 41 , não apresentou diferenças significativas entre os grupos com sobressaliência, com sobremordida ou com ambas más oclusões, ficando o valor médio das duas categorias dentro da normalidade. A associação das duas más oclusões não representou alteração na profundidade de sondagem, com uma média em torno de $2,4 \mathrm{~mm}$.

\section{CONCLUSÕES}

Diante dos resultados obtidos, esse estudo permite concluir que:

1) Houve uma tendência a um maior acúmulo de placa nos pacientes portadores de sobressaliência e sobremordida $>6 \mathrm{~mm}$ associadas e nos portadores de mordida cruzada posterior, principalmente quando apenas um elemento dentário se encontrava nesta condição (16 ou 26).

2) Houve uma tendência à maior profundidade de sondagem nos pacientes portadores de mordida cruzada posterior, mas sem significado estatístico.

3) Não foram detectadas alterações periodontais significantes nos traços de má oclusão avaliados na população estudada. 


\title{
Periodontal evaluation of patients between 10 and 18 years with different malocclusion
}

\begin{abstract}
Aim: to evaluate the periodontal status of 41 patients with ages between 10 and 18 years. Methods: all the patients presented one of the following severe malocclusion: overjet greater than $6 \mathrm{~mm}$, overbite greater than $6 \mathrm{~mm}$, posterior unilateral or bilateral cross-bite or overjet and overbite greater than $6 \mathrm{~mm}$. The periodontal indexes of plaque and bleeding and the parameters of probing depth, amount of keratinized mucosa, periodontal recession and attachment level were measured in index teeth $(16,26,36,46,11$ and 42). The data collected was evaluated by the Kruskall Wallis statistical test $(p<0.05)$. Results: periodontal of the teeth in the four groups of malocclusion was within the normal limits. However, the group with posterior cross-bite as well as the group with severe overjet and overbite associated, demonstrated tendency to have a greater plaque index when compared to normal parameters. The group, with posterior cross-bite malocclusion, also showed a tendency to have deeper probing depth, but without statistic significance. Conclusion: the severity of malocclusion was not significantly related with the presence of periodontal disease.
\end{abstract}

Key words: Malocclusion. Periodontal disease.

\section{REFERÊNCIAS}

1. ADDY, M.; GRIFFITHIS, G. S.; DUMMER, P. M. H. The association between tooth irregularity plaque accumulation, gingivitis and caries in 11-12 year-old children. Eur J Orthod, London, v. 10 , no. 4, p. 76-83, Oct. 1988.

2. AINAMO, J.; PALOHEIMO, L.; NORDBLAD, A.; MURTOMAA, $\mathrm{H}$. Gingival recession in school children at 7,12 and 17 years of age in Espoo, Finland. Community Dent Oral Epidemiol, Copenhagen, v. 14, p. 283-286, 1986

3. ALEXANDER, A. G.; TIPNIS, A. K. The effect of irregularity of teeth and the degree of overbite and overjet on the gingival health. Br Dental J, London, v. 128, p. 539-544, June 1970.

4. AL-JASSER, N.; HASHIM, H. Periodontal findings in cases of incisor cross-bite. J Clin Pediatr Dent, Birmingham, v. 19, no. 4, 285-287, Apr. 1995.

5. BJORNAS, T.: RYGH, P.; BOE, O. E. Severe overiet and overbite reduced alveolar bone height in 19-year old men. Am J Orthod Dentofacial Orthop, St. Louis, v. 106, no. 2, p. 139-145, Aug. 1994.

6. BUCKLEY, L. A. The relationship between malocclusion and periodontal disease. J Periodontol, Chicago, v. 43, p. 415-417, July 1972.

7. GEIGER, A. M. Oclusal studies in 188 consecutive cases of periodontal disease. Am J Orthod, St. Louis, v. 48, no. 5, p. 330-359, May 1962

8. GEIGER, A. M.; WASSERMAN, B. H.; TURGEON, L. R. Relationship of occlusion and periodontal disease. Part VI. Relation of anterior overjet and overbite to periodontal destruction and gingival inflammation. J Periodontol, Chicago, v. 44, no. 3, p.150-157, Mar. 1973

9. GLICKMAN, I. Clinical significance of trauma from occlusion. J Am Dent Assoc, Chicago, v. 70, p. 607-618, Mar. 1965.

10. GOULD, M. S. E.; PICTON, D. C. A. The relation between irregularities of the teeth and periodontal disease. Br Dental J, London, v. 121, p. 20-25, July 1966.

11. HELM, S.; PETERSEN, P. E. Causal relation between malocclusion and periodontal health. Acta Odontol Scand, Oslo, v. 47, p. 221-228, June 1989
12. HÖRUP, N.; MELSEN, B.; TERP, S. Relationship between malocclusion and manteinance of teeth. Community Dent Oral Epidemiol, Copenhagen, v. 15, p. 74-78, July 1987.

13. LÖE, H. The gingival index, plaque index and the retention index system. J Periodontol, Chicago, v. 38, p. 626, 1967.

14. MILLER, J.; HOBSON, P. The relationship between malocclusion, oral cleanliness, gingival conditions and dental caries in school children. Br Dental J, London, v. 111, p. 43-52, Apr. 1961.

15. POULTON, D. R.; AARONSON, J. A. The relationship between occlusion and periodontal status. Am J Orthod, St. Louis, v. 47 p. 690-699, 1961

16. QUIGLEY, G., HEIN, H. Comparative cleansing efficiency of manual and power brushing. J Am Dental Assoc, Chicago, v. 65 p. 26-29, July 1962.

17. SOCRANSKY, S. S.; HAFFAJEE, A. D. The bacterial etiology of destructive periodontal disease. J Periodontol, Chicago, v. 63, p. 322-331, 1992.

18. SOLBERG, W. C. The role of morphofunctional occlusal factors in periodontal disease. In: CARRANZA, F. A. (Ed.). Glickman's Clinical Periodontology. 7 th ed. Philadelphia: W. B. Saunders, 1990. p. 422-431.

19. RAMFJORD, S. P. Indexes for prevalence and incidence of periodontal disease. J Periodontol, Chicago, v. 30, p. 51-59, 1966.

\author{
Endereço de correspondência \\ Elton Gonçalves Zenóbio \\ Pontifícia Universidade Católica de Minas Gerais - PUC Minas \\ Mestrado em Ortodontia \\ Rua Don José Gaspar 500 - Prédio 46 \\ CEP: 30.535-610 - Belo Horizonte/MG \\ E-mail: daniela.soarescorrea@gmail.com
}

